psoriasis and atopic dermatitis in order to increase effectiveness of dermatological treatment.

Method. 59 patients with atopic dermatitis, 67 with psoriasis and 65 healthy control group individuals were included in the crosssectional study. Predominant complains of the patients: itching, widespread rashes and rashes on the open areas of the skin. In $85 \%$ patients with skin pathology onset of the disease and relapses were associated with stress, in $15 \%$ other factors.

"The 20-item Toronto Alexithymia Scale" was used to assess alexithymia. "The Ways of Coping Checklist, Lazarus" was used to assess coping-strategies. "The Holmes and Rage Stress Inventory" was used to assess stress contribution to illness. Significance level: $\mathrm{p}<0,05$.

Result. The levels of alexithymia ( $p=0.002)$, difficulty identifying feelings subscale $(\mathrm{p}=0.02)$ and externally-oriented thinking subscale $(p=0.002)$ in patients with skin pathology (especially in those with psoriasis) were higher than in the control group.

Patients with skin pathology turned out to be more susceptible to stress factors $(\mathrm{p}=0.025)$ and less often use coping strategy "seeking social support" $(\mathrm{p}=0.037)$.

Patients with skin pathology with high levels of alexithymia and difficulty identifying feelings subscale more likely to use maladaptive "escape-avoidance" coping $(\mathrm{p}=0.001)$.

Patients with atopic dermatitis who find difficult to describe feelings are more likely to use maladaptive coping "distancing" $(p=0.002)$.

In patients with psoriasis high levels of alexithymia and externally-oriented thinking subscale scores are associated with less common use of the adaptive coping "problem solving" $(p=0.001)$. Moreover, in patients with psoriasis high levels of difficulty identifying feelings subscale are associated with more common use of maladaptive "escape-avoidance" coping $(\mathrm{p}=0.001)$.

Conclusion. The results of the study confirm the need to include psychological assessment and psychotherapy in the treatment plan for patients with psoriasis and atopic dermatitis in order to improve emotional awareness and to develop more adaptive coping-strategies in patients.

\section{Predicting risks of physical health deterioration in a place of safety}

Alex Berry ${ }^{1 \star}$, Florence Dalton ${ }^{2}$, Michael Dunning ${ }^{2}$ and Freddie Johansson ${ }^{2}$

${ }^{1}$ National Hospital for Neurology and Neurosurgery and ${ }^{2}$ Camden and Islington Foundation NHS Trust

${ }^{*}$ Corresponding author.

doi: 10.1192/bjo.2021.81

Aims. Healthcare triage for those subject to section 136 powers (MHA 1983/2007) remains challenging. Camden and Islington NHS Foundation Trust opened a dedicated Health-Based Place of Safety (HBPOS) in 2020, situated separately from an emergency department (ED). There was concern that this may lead to physical health problems going unrecognised. We aimed to design a simple, efficient algorithm to be used by non-medically-trained staff to identify those who are subject to s.136 powers who would benefit from medical clearance before being admitted to the HBPOS

Method. We chaired a consensus meeting with nursing staff, police and emergency medicine consultants when designing the algorithm. Case notes of those presenting under s.136 to the POS over 1 calendar-month in 2019 were reviewed, and the proportion of those who the algorithm would have diverted for medical clearance was calculated. We then reviewed the proportion of cases sent for medical clearance during a single calendar month in
2020, after the HBPOS had opened, to see whether there was a significant difference.

Result. 37 patients were admitted to the ED-based POS in July 2019, of which 36 records were analysed. 9 patients (25\%) were referred for medical clearance, with $2(6 \%)$ requiring medical admission. $8.6 \%$ were identified as needing medical clearance when the algorithm was applied retrospectively (positive predictive value $66 \%$, negative predictive value $=79 \%$ ).

Review of records over 1 calendar-month after the HBPOS was established showed $30.6 \%$ of patients had been diverted for medical clearance prior to entering the HBPOS. Of the 65 patients, 1 (2\%) required transfer to ED within 48 hours of entry. No statistical difference in the proportion of patients sent for medical clearance was observed since the formation of the HBPOS away from the ED (Chi-squared $=0.549, \mathrm{p}=0.458$ ), suggesting the algorithm successfully identified those patients who needed medical clearance prior to admission.

We observed high rates of intoxication amongst those admitted $(30-40 \%)$.

Conclusion. The algorithm showed high specificity and negative predictive value, allowing for a degree of confidence when admitting those deemed at low-risk of physical deterioration, though it does not eliminate the need for clinical judgement. Interpretation of the results is complicated by the COVID19 pandemic in 2020, which was not accounted for in the algorithm, which possibly led to deviations from the algorithm in real-world clinical practice.

Reflections on a person's experience of mental illness: an innovative teaching pilot for second-year medical students

George Blanchard ${ }^{1 *}$, Louis Quail ${ }^{2}$, Grace Yang ${ }^{2}$,

Katherine Terence ${ }^{2}$, Amisha Kalra ${ }^{2}$, Neil Sarkar ${ }^{3}$, Aimee Spector ${ }^{4}$, Seri Abraham ${ }^{5}$ and Suzanne Reeves ${ }^{6}$

${ }^{1}$ UCL Medical School, Camden and Islington NHS Foundation Trust; ${ }^{2}$ UCL Medical School; ${ }^{3}$ Camden and Islington NHS Foundation Trust; ${ }^{4}$ University College London; ${ }^{5}$ Manchester Metropolitan University, Health Education North West, Royal Oldham Hospital, Oldham, Pennine Care NHS Foundation Trust and ${ }^{6}$ University College London, Camden and Islington NHS Foundation Trust ${ }^{*}$ Corresponding author.

doi: 10.1192/bjo.2021.82

Aims. We sought to develop a teaching pilot to help year 2 medical students meet the following learning outcomes: Develop a better understanding of patient and carer experiences of mental illness; Recognise and challenge unhelpful attitudes towards people with mental illness; Promote a broader understanding of cultural issues surrounding mental illness, including stigma and discrimination.

Method. 337 medical students were invited to attend a lecture by author LQ, a documentary photographer who presented a narrative of his brother Justin's lived experience of schizophrenia (louisquail.com/big-brother-introduction). 197 students attended the session, which was recorded and made available online. Students were invited to enter a competition to win a signed copy of LQ's book, 'Big Brother' and asked to submit either a 500 -word written reflective piece, or a creative work accompanied by a 200-word statement. 13 submissions were received, including paintings, drawings, collage, photography, and poetry, all of which were blind rated by authors SR and GB, based on originality and quality of reflection. Of the six shortlisted, three winning entries were chosen by author LQ. 